\section{Avaliação crítica do Sistema de Informação da Atenção Básica (SIAB) e de sua implantação na região de Ribeirão Preto, São Paulo, Brasil}

\author{
Critical evaluation of the Primary Care \\ Information System (SIAB) and its implementation \\ in Ribeirão Preto, São Paulo, Brazil
}

Anderson Soares da Silva ${ }^{1}$

Milton Roberto Laprega ${ }^{1}$

\section{Introdução}

As the health information system used by the Family Health Program teams, the Primary Care Information System (SIAB) is a potential tool for follow-up of registered families and local planning, thus motivating the present study. Semistructured interviews were held with key individuals in this health information system chain, among Family Health Teams in the municipality of Ribeirão Preto and surrounding region, São Paulo State, Brazil, with the objective of analyzing certain characteristics in the system, including: knowledge and having received training to handle it and utilization for local planning and social control. The study concluded that the SIAB is an easy system to handle, but that it presents some limitations already found in other health information systems, such as: difficulty in identifying individuals in the program, limited number of diseases reported, and limited utilization to back planning and decision-making at the local level. Therefore, the analysis of the SIAB was highly important for improving this health information system and can contribute to future improvements and adaptations in the program.

Information Systems; Program Evaluation; Family Health Program

\section{Características dos sistemas} de informação em saúde no Brasil

A Constituição Federal de 1988 e a Lei n. 8.080/ 90 (Diário Oficial da União 1990; 19 set) lançaram as bases para a criação do Sistema Único de Saúde (SUS), o qual passou a reunir todos os serviços públicos (federal, estadual e municipal) e privados (conveniados/contratados) de saúde, pregando o acesso universal e equânime ao sistema, bem como enfatizando ações de promoção de saúde, prevenção, tratamento e reabilitação de doenças.

Com a consolidação da implantação do SUS, houve a necessidade de uma melhor estruturação dos Sistemas de Informação em Saúde (SIS), para que os mesmos seguissem a lógica do acompanhamento integral pregada pelo novo sistema de saúde, assegurando a avaliação permanente da situação de saúde da população e dos resultados das ações executadas, fundamental para o acompanhamento, controle e repasse de recursos 1 . Desta forma, os municípios passaram a ser também responsáveis pela produção, organização e coordenação das informações em saúde, devendo deixar de lado o mero papel de coletor e repassador de dados. Contudo, sabe-se que grande parte dos SIS federais foi concebida antes da implantação do SUS, não incorporando, desta maneira, 
as características exigidas pelo novo sistema de saúde (por exemplo: a descentralização das informações).

Pode-se, assim, resumir as principais características dos SIS em funcionamento no Brasil, segundo Moraes 2 e Tasca et al. 3: (a) centralizados/verticalizados/fragmentados - os dados obedecem ao fluxo municípios-estados-federação, ou seja, sempre na direção do nível local para o central, são fragmentados, pertencendo aos vários feudos técnicos da saúde e sofrem processamento fundamentalmente no nível central; (b) exclusão do nível local (municípios) no processo de decisão e planejamento em saúde; (c) ausência de mecanismos de avaliação e controle da qualidade dos dados produzidos; (d) ênfase na coleta de dados médicos ou de doenças, não permitindo a construção do perfil de saúde da população; (e) incompatibilidade entre os diversos sistemas de informação utilizados; (f) falta/deficiência de infraestrutura de informática nos municípios, o que dificulta ou até mesmo inviabiliza a coleta adequada e o processamento dos dados; (g) ter como base dos dados apenas a população assistida, o que contribui para a produção de informações não compatíveis com a realidade local; (h) ausência da participação popular na geração e uso das informações.

Então, fundamentalmente, o que tem ocorrido é apenas a descentralização da digitação, processamento e acesso aos dados, ficando ainda às instâncias centrais (estadual e federal) a definição das prioridades a serem seguidas 4 .

\section{Sistema de Informação da Atenção Básica}

O Sistema de Informação da Atenção Básica (SIAB) foi criado em 1998 pelo Departamento de Informação e Informática do SUS (DATASUS), em conjunto com a Coordenação de Saúde da Comunidade/Secretaria de Assistência à Saúde (COSAC/SAS), para auxiliar o acompanhamento e avaliação das atividades realizadas pelos agentes comunitários de saúde (ACS), agregando e processando os dados advindos das visitas domiciliares, bem como, do atendimento médico e de enfermagem realizado na unidade de saúde e nos domicílios 5 .

É composto por um programa de computador (software) e por algumas fichas (A, B, C, D) e relatórios (SSA-2, SSA-4, PMA-2, PMA-4 e A1 ao A4). A ficha A representa a ficha de cadastro familiar e, portanto, contém dados básicos de características sócio-econômicas, de saúde (morbidade referida) e moradia das famílias e seus indivíduos. As fichas B são utilizadas pelos ACS para o acompanhamento domiciliar de grupos prioritários, como: hipertensos, diabéticos, gestantes, hansenianos e tuberculosos. A ficha C presta-se ao acompanhamento das condições de saúde e seguimento médico de crianças menores de dois anos, sendo o próprio Cartão da Criança fornecido pelo Ministério da Saúde. A ficha D é utilizada por toda a equipe do Programa Saúde da Família (PSF) para o registro das atividades diárias (consultas médicas e de enfermagem, solicitação de exames complementares, encaminhamentos), bem como para a notificação de algumas doenças (por exemplo: pneumonia em menores de cinco anos). Já os relatórios representam um consolidado dos dados presentes nas fichas de cadastro e acompanhamento: (1) SSA2 - consolidado dos dados das fichas A, B, C e D; (2) SSA4 consolidado dos dados contidos nos relatórios SSA2 de um município; (3) PMA2 - consolidado das fichas D; (4) PMA4 - consolidado dos relatórios PMA2 do município; (5) relatórios A1 ao A4 - consolidado dos dados presentes nas diversas fichas A. Os números 1, 2, 3 e 4 referem-se aos níveis de agregação correspondente: 1 - micro-área, 2 - área, 3 - seguimento e 4 município.

Quanto ao software SIAB, o mesmo utiliza três formulários de entrada dos dados: um para o cadastramento familiar, um para as informações de saúde e outro para as informações de produção e marcadores para avaliação.

Diferentemente de outros sistemas de informação em saúde, o SIAB caracteriza-se por ser um SIS territorializado, ou seja, fornece indicadores populacionais (morbidade, mortalidade e de serviços) de uma determinada área de abrangência. Propõe, com isso, que se conheçam as condições de saúde dessa população adscrita, bem como os fatores determinantes do processo saúde-doença. Representa, então, potencialmente, uma fonte de dados de grande valor para a realização do diagnóstico de saúde de determinada área de abrangência, norteando o planejamento e avaliação de ações em saúde.

\section{Métodos}

Este trabalho teve como base metodológica da construção e análise dos dados a pesquisa qualitativa.

Para a coleta dos dados, optou-se pela realização de entrevistas semi-estruturadas, individuais e em grupo, com os indivíduos-chave no manejo do SIAB, desde o nível local (equipes do PSF), passando pelas respectivas Secretarias Municipais de Saúde (SMS), até o nível 
central (Direção Regional de Saúde - DIR XVIII). Quanto à adequação dessa técnica de pesquisa para o estudo em questão, Ziebland \& Wrigtht 6 (p. 113) justificam: "técnicas de entrevista qualitativa podem ser utilizadas para explicitar pontos de vista e opiniões do público em geral, usuários e provedores de serviços, e aqueles ligados à realização de políticas e implementação de processos".

Por conseguinte, foram realizadas entrevistas em grupo com os ACS e entrevistas individuais com as respectivas enfermeiras das equipes e com os responsáveis técnicos pelo SIAB nas SMS e na DIR XVIII. Vale salientar que, antes de iniciar as entrevistas, a importância e os objetivos do estudo foram explicitados para os entrevistados e aos mesmos foi pedido, caso concordassem com a pesquisa, que assinassem um termo de consentimento.

Desta forma, foram escolhidos cinco Núcleos de Saúde da Família (NSF) no Município de Ribeirão Preto, São Paulo, Brasil, sendo esses ligados ao Centro de Saúde Escola Dr. Joel D. Machado, Faculdade de Medicina de Ribeirão Preto, Universidade de São Paulo e escolhidos aleatoriamente outros quatro Núcleos pertencentes a municípios ligados à DIR XVIII (uma equipe em Serrana e Jardinópolis, e duas em Luiz Antônio). Quanto aos técnicos responsáveis pelo SIAB nas SMS, foram apenas entrevistados aqueles dos Municípios de Ribeirão Preto e Serrana, visto que, nos demais municípios, não havia pessoal encarregado para tal função.

Para a escolha dos outros municípios pertencentes à DIR XVIII, foi dada preferência àqueles que tinham duas ou mais equipes de saúde da família, e, dentre essas, foram escolhidas aquelas com maior tempo de funcionamento.

As entrevistas foram realizadas entre os meses de julho e setembro de 2002, com duração média de uma hora, perfazendo um total de 19 entrevistas, sendo os sujeitos da pesquisa (enfermeiras, ACS e responsáveis técnicos pelo SIAB) entrevistados em seus locais de trabalho.

Quanto à análise do material obtido (entrevistas), o primeiro passo foi a transcrição das mesmas. Feito isso, o próximo passo foi a leitura exaustiva de todas as transcrições, objetivando-se entender o fenômeno sob investigação. Essa etapa da análise também auxiliou, em muito, a construção da próxima, qual seja, a análise de conteúdo do material.

A análise de conteúdo representa um dos métodos mais comuns de análise dentro da metodologia qualitativa. Quanto a esse ponto, os autores Ziebland \& Wright 6, Knodel et al. 7 e
Bender \& Ewbank 8 concordam: representa o exame sistemático dos textos e notas de entrevistas, identificando e agrupando-os em temas, e classificando/desenvolvendo categorias. As categorias ou temas surgem a partir do referencial teórico e dos roteiros empregados nas entrevistas, ou quando algumas citações dos entrevistados passam a ser relevantes dentro da análise (aparecem com maior freqüência). Desta forma, a construção dos temas levou em consideração os aspectos gerais a respeito do SIAB (no contexto do estudo), os quais surgiram a partir de uma convergência dos objetivos do estudo (baseados na literatura consultada) e das falas dos entrevistados, chegando-se à seguinte divisão: (1) características do softwareentram nessa categoria as opiniões a respeito do manejo do programa de computador, dificuldades encontradas e sugestões para sua melhoria; (2) rotina de preenchimento das fichas e relatórios - nessa, estão representadas as opiniões quanto ao entendimento dos campos (com as respectivas dúvidas e sugestões) presentes nas fichas e relatórios, adaptação local às dificuldades encontradas e opiniões a respeito do manual técnico do SIAB; (3) SIAB no processo decisório - estão representados aqui o uso do SIAB como ferramenta para a realização de diagnósticos de saúde, planejamento de ações, controle social, existência de supervisão local e relações com outros SIS; (4) relação entre os diferentes níveis - nessa categoria, entram as proposições quanto à existência, ou não, de supervisão e treinamento das equipes pelas SMS e DIR XVIII, conhecimento geral e utilização do sistema pelos níveis centrais e retorno dos dados para as equipes locais.

Conforme sugerido por Bender \& Ewbank 8 e Knodel et al. 7, como forma de facilitar a análise interpretativa do conteúdo dos transcritos, foi construída uma tabela ("rede de visualização"), contendo os tópicos chaves (categorias ou temas) segundo os grupos de entrevistados. Cada célula da tabela continha sumários do conteúdo das discussões de cada grupo de acordo com cada tema ou categoria.

Assim dispostos os dados em categorias analiticamente úteis, procedeu-se a procura de características comuns existentes entre essas subdivisões, o que auxiliou a elaboração das conclusões a respeito de cada categoria.

Como forma de avaliar a validade dos dados coletados, procedeu-se a comparação das declarações entre os diversos grupos (ACS $\mathrm{x}$ ACS, enfermeiras x ACS e enfermeiras $x$ enfermeiras) ${ }^{8}$. Desse modo, pôde-se chegar a um ponto de saturação tal que conferiu validade e representatividade ao trabalho 6 . 


\section{Resultados}

Analisando o conteúdo das falas dos entrevistados intra e inter grupos, pôde-se chegar às seguintes conclusões sobre o SIAB, na região de Ribeirão Preto, na época em que o estudo foi realizado:

\section{Características gerais do software}

Todos ressaltaram a simplicidade de manuseio do programa, porém criticaram a impossibilidade de digitar o nome das pessoas e os respectivos endereços, fato esse que facilitaria a melhor identificação das famílias e dos indivíduos dentro do programa.

Outra característica negativa bastante ressaltada nos dois grupos (ACS e enfermeiras) foi o número limitado de doenças ou condições referidas codificadas na ficha $\mathrm{A}$, os quais não refletiriam a realidade local. Como exemplo, foi citada a impossibilidade de registrar doenças como depressão e AIDS, em detrimento de outras codificáveis pelo SIAB, mas de menor prevalência ou até mesmo inexistentes para a maioria das equipes (e.g. malária).

O número limitado de ocupações que o programa oferece também foi muito citado, ficando os ACS obrigados, em muitos casos, a adaptar a classificação da profissão de determinado indivíduo àquela existente no programa.

Outro fato notado pela maioria das equipes apenas depois de certo tempo de utilização do software foi a não mudança automática da idade dos indivíduos cadastrados após fazerem aniversário, acarretando a contabilização errada dessa população nos relatórios de produção, gerando confusão para os membros das equipes.

\section{Preenchimento das fichas e relatórios}

A maioria dos profissionais das equipes, principalmente os ACS, reclamou do excesso de fichas usadas em sua rotina de trabalho, o que resultaria em perda de tempo, dificultando a dinamização do trabalho.

Algumas enfermeiras também se queixaram do manual técnico do SIAB, classificandoo de "superficial e pontual", pois não continha respostas às principais dúvidas sobre o SIAB, bem como não fornecia informações sobre como utilizar melhor todos os recursos que o sistema dispõe.

Quanto à ficha de acompanhamento das gestantes (ficha B-GES), a maioria das enfermeiras referiu haver dúvidas no seu preenchimento pelos ACS, sendo a dificuldade técnica em determinar o estado nutricional da gestante o fator mais citado, ponto esse também mencionado pelos ACS.

Sobre a ficha de acompanhamento dos hipertensos (ficha B-HA), os campos que mais geraram dúvidas foram os da pressão arterial e dieta. Sobre este, as enfermeiras revelaram-se preocupadas com a orientação dada pelos ACS durante as visitas domiciliares, pois haveria a necessidade de treinamento desses profissionais para que pudessem reforçar as orientações dadas aos pacientes hipertensos pelos médicos e enfermeiras sobre os cuidados com a alimentação, em seu domicílio. Já aquele foi classificado como de pouca confiabilidade, visto que, segundo o Conselho Regional de Enfermagem do Estado de São Paulo, aos ACS não é permitido aferir a pressão arterial, e também pela dificuldade dos pacientes em lembrar o último valor da pressão arterial aferido.

A ficha de acompanhamento dos portadores de diabetes (ficha B-DIA) foi caracterizada pelas equipes como aquela de mais fácil preenchimento. Considerações foram feitas por algumas enfermeiras a respeito do campo: dieta e exercício. Para elas, os ACS deveriam ser adequadamente orientados, de forma semelhante à observação feita para a ficha B-HA, como indagar e orientar os pacientes sobre alimentação saudável e atividade física durante as visitas domiciliares.

Ainda sobre as fichas de acompanhamento de hipertensos e diabéticos, alguns profissionais entrevistados reclamaram da não utilização pela equipe dos dados coletados, o que pode ser exemplificado na fala deste ACS: "eu faço isso, mas o médico nunca pega essa ficha para ver, então para quê? É um papel a mais, porque o médico não pega essa ficha".

As fichas de acompanhamento dos portadores de tuberculose (B-TB) e hanseníase (BHAN) praticamente não eram utilizadas, devido à baixa prevalência desses casos nas áreas de abrangência das equipes e/ou ao acompanhamento desses pacientes pelos profissionais da vigilância epidemiológica local.

A ficha C não era utilizada conforme orientação do manual. Praticamente cada equipe criou a sua própria ficha, quase todas acrescentando, além do calendário vacinal, outros campos contendo dados sobre seguimento de puericultura, peso, tipo de aleitamento e ocorrência de diarréia, infecção respiratória aguda ou uso de terapia de reidratação oral no mês.

Quanto à ficha de registro de atividades, procedimentos e notificações (ficha D), essa não era usada pelos ACS, por acharem-na desnecessária (alegaram que seria duplicação de 
trabalho, pois os dados que essa ficha contém são todos passados para o software SIAB). Era utilizada, sim, pelos médicos e enfermeiras, sendo que estas se queixaram da existência de alguns problemas, tais como: inadequação da definição de procedimentos coletivos (deixaria de fora atividades como participação em programas de rádio comunitária, grupos de atividade física) e impossibilidade de registrar melhor os atendimentos do enfermeiro e do auxiliar.

Algumas equipes utilizavam o relatório SS2 (relatório de situação de saúde e acompanhamento das famílias na área) original do SIAB, porém havia outras que trabalhavam com um relatório bem semelhante, salvo algumas modificações (e.g. no campo correspondente às micro-áreas, havia os dias do mês). Consideraram-no de fácil preenchimento, porém trabalhoso. Algumas enfermeiras e ACS referiram não confiar na validade do campo: nutrição da criança, devido à incapacidade técnica dos ACS em saber avaliar essa parte, visto que, em apenas um dos NSF, os ACS pesavam as crianças no domicílio.

Também o relatório de produção e marcadores para avaliação (PMA2) era pouco utilizado pelas equipes, sendo seus dados digitados diretamente no programa de computador. Alguns declararam sempre deixar em branco os campos referentes aos marcadores, e sobre esse fato é digna de nota a observação de um enfermeiro: "desde o nosso trabalho, eu senti falta de alguns marcadores..., outros marcadores que refletem a nossa realidade; as doenças respiratórias seriam aqui um bom marcador".

\section{SIAB no processo decisório}

Em praticamente todas as equipes, a supervisão do preenchimento das fichas e relatórios era feita de maneira informal, ocorrendo ocasionalmente durante a conclusão dos relatórios do SIAB a serem enviados à SMS, realizado no final de cada mês. Quanto às dúvidas que surgiam, os ACS procuravam as enfermeiras no dia-a-dia de trabalho ou em espaços reservados durante a semana para a discussão de casos pertinentes às famílias.

Evidenciou-se também que, em todas as equipes do PSF estudadas, não havia o repasse ou discussão com a comunidade dos dados gerados pelo sistema, ou seja, inexistia a participação comunitária no planejamento e na tomada de decisões.

Quanto ao emprego do SIAB como instrumento de planejamento em saúde no nível local, as equipes pouco utilizavam seus recursos para esse fim. Sua maior utilização visava ape- nas ao levantamento numérico de algumas condições de saúde/doença (por exemplo: número de hipertensos, gestantes, diabéticos), para a realização de grupos de saúde com a comunidade.

Ademais, foi identificada a ocorrência de outros SIS utilizados pelas equipes, tais como os sistemas: HIPERDIA (Sistema de Cadastramento e Acompanhamento de Hipertensos e Diabéticos), HYGIA (utilizado para registro de produção, marcação de consultas e exames laboratoriais), SISVANSP (Sistema de Vigilância Alimentar e Nutricional do Estado de São Paulo) e SIA/SUS (Sistema de Informações Ambulatoriais do SUS), todos eles empregados com uma finalidade para a qual o próprio SIAB poderia servir.

\section{Relação entre os níveis}

No período do estudo, todas as equipes do PSF de Ribeirão Preto e aquelas pertencentes aos municípios entrevistados utilizavam o sistema SIAB em sua versão informatizada.

Quanto à existência de responsáveis técnicos pelo SIAB, esse fato pôde ser constatado somente na DIR XVIII e nas SMS de Ribeirão Preto e Serrana. Porém, nesses dois municípios, esses técnicos tinham como função apenas consolidar os dados do município e enviálos, mensalmente, à equipe técnica da DIR.

Os níveis regionais foram unânimes em confirmar a regularidade de envio dos relatórios e dados consolidados pelas equipes locais, porém, as SMS funcionavam apenas como repassador dos dados, e a DIR realizava somente a análise da cobertura e checava possíveis erros de digitação ou consistência nos dados consolidados e relatórios (por exemplo: número de famílias cadastradas $\mathrm{x}$ acompanhadas).

Como não havia análise dos dados pelas SMS ou DIR, os dados também não retornavam às equipes locais do PSF.

Todas as equipes locais receberam treinamento para lidar com o SIAB, no caso das equipes de Ribeirão Preto, sob a responsabilidade da SMS, e as equipes dos outros municípios, pela DIR XVIII. Porém, o treinamento não foi suficiente para sanar todas as dúvidas existentes, pois, segundo uma enfermeira, foi direcionado somente para os recursos que o SIAB oferece para o envio dos relatórios às secretarias municipais (as fichas A e B, e relatórios PMA2 e SSA2), deixando de fora outros recursos que o programa pode fornecer.

Quanto aos outros níveis, os funcionários da DIR receberam treinamento da equipe técnica do PSF/PACS da Secretaria Estadual de 
Saúde (Coordenadoria de Saúde do Interior CSI), no entanto o mesmo não aconteceu com a equipe da SMS de um dos municípios, que, de acordo com a enfermeira responsável pelo SIAB na época da implantação das equipes do PSF, referiu: "eu não tive treinamento nenhum. Simplesmente li o manual do Ministério da Saúde e fui tentando ajudar as equipes a preencher a ficha $A$, as fichas $B$ e depois juntar todos os dados para poder encaminhar para a DIR. No começo, foi muito difícil. A gente não tinha idéia de como fazer".

\section{Discussão}

Com a realização deste trabalho, conclui-se que o SIAB, da forma como está estruturado e é utilizado pelas equipes do PSF, SMS e DIR, nos municípios analisados e no período em que o estudo foi feito, possui várias características importantes para um SIS voltado para atenção básica, porém apresenta alguns problemas de ordem estrutural e de aplicação, que em muito se assemelham aos demais SIS em funcionamento no país.

Apesar da simplicidade do programa, permitindo que pessoas sem qualquer treinamento em informática o manuseie, as falhas encontradas no software (e.g. não identificação de indivíduos, pequeno número de doenças codificáveis) podem distorcer o retrato que ele fornece da população adscrita. Para tanto, sugerese, como forma de facilitar a localização de indivíduos e famílias, mudanças nos indicadores de cadastramento familiar, permitindo que o software contenha a identificação dos indivíduos cadastrados, unificando-o com o cadastro do cartão SUS 9, a adoção da tabela da Classificação Brasileira de Ocupações como tentativa de resolução da falta de ocupações codificáveis no SIAB 10 e a ampliação do número de doenças codificáveis no software, de acordo com a realidade da Atenção Primária, baseando-se nas doenças e agravos mais comuns desse nível de atenção, podendo-se utilizar, como base, formas de codificações internacionais já bem estabelecidas 11 .

Há também a necessidade de o Ministério da Saúde melhorar o manual do SIAB, enriquecendo-o com explicações mais detalhadas sobre o preenchimento das fichas e a operacionalização do software (obtenção de relatórios, marcadores etc.), sugestões essas já feitas em documento oficial para outros SIS 12.

No que se refere às fichas e aos relatórios, devido às várias adaptações que alguns desses instrumentos sofreram e a não utilização de outros (ficha C, relatório SSA2, relatório PMA2), há que se discutir possíveis reformulações, para que melhor se adaptem ao trabalho das equipes do PSF, melhorias essas que devem ser fruto da interlocução entre todos (equipes locais e gestores) os envolvidos no manejo do SIAB 13. Sugere-se, então, a adoção de uma ficha C padrão, semelhante às fichas $\mathrm{B}$, que contenha dados importantes para o acompanhamento das crianças até dois anos, tais como: tipo de aleitamento, vacinas, peso, principais intercorrências; a reformulação da ficha $\mathrm{D}$, melhorando o registro do trabalho dos enfermeiros e auxiliares de enfermagem; e a reformulação dos marcadores presentes no relatório PMA2, para que melhor reflitam a realidade da Atenção Primária, por exemplo, utilizando-se de marcadores para os quais existam programas ou aqueles que pudessem utilizar os dados já disponíveis na unidade de saúde ${ }^{9}$, bem como acrescentar campos para registro de ações de prevenção de doenças e promoção de saúde (Comissão Intergestores Tripartite. Reunião em 21/11/2002. http://intranet.ensp.fiocruz.br/descentralizar/print_debates.cfm?debate $=58 \& \mathrm{txt}=212$, acessado em 31/Jan/2003), participação comunitária e colaboração intersetorial 14 . Ademais, chama a atenção o fato de as fichas B não serem adequadamente aproveitadas pelas equipes, segundo relato dos ACS, principalmente pelos médicos, que também pouco conhecem ou utilizam o SIAB. Essa é uma preocupação realmente pertinente desses profissionais, pois essas fichas contêm dados de fundamental importância para o acompanhamento dos indivíduos cadastrados, ou seja, deveriam ser objeto de discussão e intervenção por parte da equipe 15 .

Apesar de ser um SIS territorializado, o SIAB ainda é verticalizado e centralizado, ou seja, o fluxo obedece à direção do nível local para o central, e a análise dos dados ainda se faz fundamentalmente no nível central (Ministério da Saúde). Cometem-se, desta forma, os mesmos erros de outros SIS, onde a análise dos dados se faz principalmente no nível central, ficando os níveis local e regional como meros repassadores de dados. Perdem, então, as SMS e as equipes locais, pois, sendo o SIAB um SIS territorializado, possibilita, por meio dos seus indicadores, a micro-localização de problemas 16, fato esse de valor inestimável para o planejamento e a tomada de decisão nos níveis local/regional.

Quanto à falta de supervisão e controle da qualidade dos dados produzidos pelas equipes do PSF, esse fato compromete sobremaneira a própria confiabilidade das informações geradas 2 . Ou seja, a adequada supervisão das fichas e relatórios realmente mereceria maior 
atenção por parte das equipes locais, pois a qualidade do registro dos dados é parte fundamental de qualquer SIS.

Também, a constatação de incompatibilidade da plataforma do SIAB com outros SIS (SIA/SUS, HYGIA, HIPERDIA) utilizados pelas equipes resultava em duplicação dos dados produzidos, ocasionando perda de tempo e de recursos financeiros.

Não foi evidenciado, por parte das equipes locais, o adequado estímulo à participação da comunidade na geração e uso das informações para a tomada de decisão. Tornar as informações em saúde transparentes para a população é parte fundamental do processo de democratização da tomada de decisões em nível local. É parte importante da construção da cidadania 17. Não fazendo isso, as equipes do PSF, enquanto serviços de Atenção Primária, estão deixando de desempenhar seu importante papel na democratização do SUS.

Por fim, o SIAB constitui-se em um instrumento fundamental para a gestão das unidades do PSF, que poderia ter sua importância aumentada caso ocorressem algumas melhorias no software, nas fichas e relatórios, bem como um maior conhecimento e utilização por parte das equipes locais e dos cidadãos usuários.

\section{Resumo}

Por ser o Sistema de Informação em Saúde (SIS) utilizado pelas equipes do Programa Saúde da Família (PSF), o Sistema de Informação da Atenção Básica (SIAB) representa uma ferramenta em potencial para o acompanhamento das famílias cadastradas, assim como para o planejamento local, razão pela qual se propôs o presente estudo. Foram realizadas entrevistas semi-estruturadas com os indivíduos-chave na cadeia de articulação deste SIS, em equipes do PSF do Município de Ribeirão Preto e região, São Paulo, Brasil, com o objetivo de analisar algumas características desse sistema, tais como: conhecimento, recebimento de treinamento para o seu manejo, utilização para planejamento local e controle social, dentre outros. Concluiuse que o SIAB é um sistema de fácil manipulação, mas apresenta algumas limitações já encontradas em outros SIS, como: dificuldade de identificação de indivíduos no software, número limitado de doenças referidas, pouca utilização para subsidiar o planejamento $e$ a tomada de decisões em nível local. Portanto, a análise do SIAB foi de suma importância para o aprimoramento deste SIS, podendo contribuir para futuras melhorias ou adaptações no programa.

Sistemas de Informação; Avaliação de Programas; Programa Saúde da Família
Com vistas a essas e outras mudanças necessárias, já existe, há algum tempo, um grupo do Ministério da Saúde e do DATASUS discutindo e propondo mudanças para o SIAB, objetivando torná-lo um sistema de avaliação e monitoramento capaz de refletir a complexidade e heterogeneidade das várias realidades municipais e dos diferentes níveis de gestão do SUS. Foi batizado de SIAB/PLUS e teria como objetivo primordial "disponibilizar aos gestores do SUS, nos diversos níveis, informações que reflitam o perfil de atendimento e a situação de saúde (processo assistencial e morbidade ambulatorial), permitindo adequado planejamento, acompanhamento e avaliação das ações, simplificando os processos, através da padronização, unificação, atualização tecnológica e flexibilização" (Comissão Intergestores Tripartite. Reunião em 21/11/2002). O que se espera é que, com a conclusão dos trabalhos da equipe técnica, que estava prevista para o 1o semestre de 2004 (Departamento de Informação e Informática do SUS, Ministério da Saúde. Apresentação. http://www.datasus.gov.br/siab/siab.htm, acessado em 10/Set/2004), o SIAB torne-se um SIS que realmente contribua para o monitoramento e avaliação da Atenção Básica.

\section{Colaboradores}

A. S. Silva realizou a revisão da literatura, elaborou o projeto, coletou os dados e redigiu o artigo. M. R. Laprega participou da elaboração do projeto, análise dos resultados e da redação final do artigo. 


\section{Referências}

1. Fundação Nacional de Saúde, Ministério da Saúde. Sistema de informações em saúde. Inf Epidemiol SUS 1995; 4:85-92.

2. Moraes IHS. Informações em saúde: da prática fragmentada ao exercício da cidadania. São Paulo: Editora Hucitec; 1994.

3. Tasca R, Grego C, Villarosa FN. Sistemas de informação em saúde para distritos sanitários. In: Mendes EV, organizador. Distrito sanitário: o processo social de mudança das práticas sanitárias do Sistema Único de Saúde. São Paulo: Editora Hucitec; 1995. p. 267-310.

4. Branco MAF. Sistemas de informação em saúde no nível local. Cad Saúde Pública 1996; 12:267-70.

5. Ministério da Saúde. Manual do sistema de informação da atenção básica. Brasília: Ministério da Saúde; 2000.

6. Ziebland S, Wright L. Qualitative research method. In: Jenkinson C, editor. Assessment and evaluation of health and medical care: a methods text. Buckingham/Philadelphia: Open University Press; 1997. p. 103-8.

7. Knodel J, Sittitrai W, Brown T. Focus group discussions for social science research: a practical guide with an enphasis on the topic of ageing. Ann Arbor: University of Michigan; 1990. (Population Studies Center, Research Report 90-3).

8. Bender DE, Ewbank D. The focus group as a toll for the health research: issues in design and analysis. Health Transit Rev 1994; 4:63-80.

9. Medina MG, Aquino R, Carvalho AL. Avaliação da atenção básica: construindo novas ferramentas para o SUS. Divulg Saúde Debate 2000; 21:15-28.
10. Ministério da Saúde. Compatibilização de Sistemas e Bases de Dados (CBD) da Rede Integrada de Informações para a Saúde (RIPSA) - informe final. Inf Epidemiol SUS 1997; 6:35-41.

11. Starfield B. Atenção primária: equilíbrio entre necessidades de saúde, serviços e tecnologia. Brasília: UNESCO Brasil/Ministério da Saúde; 2002.

12. Fundação Nacional de Saúde. Normatização dos sistemas de informação em saúde gerenciados pelo CENEPI. Inf Epidemiol SUS 1997; VI:20-3.

13. Moraes IHS, Santos SRFR. Informações para a gestão do SUS: necessidades e perspectivas. Inf Epidemiol SUS 2001; 10:49-56.

14. Hartz ZMA. Pesquisa em avaliação da atenção básica: a necessária complementação do monitoramento. Divulg Saúde Debate 2000; 21:29-35.

15. Nichiata LYI, Francolli LA. O Sistema de Informação da Atenção Básica - SIAB como um instrumento de trabalho da equipe no Programa Saúde da Família: a especificidade do enfermeiro. In: Instituto para o Desenvolvimento da Saúde, organizador. Manual de enfermagem. São Paulo: Ministério da Saúde; 2001. p. 29-33.

16. Carvalho DM. Grandes sistemas nacionais de informação em saúde: revisão e discussão da situação atual. Inf Epidemiol SUS 1997; VI:7-46.

17. Teixeira RR. Informação e comunicação em saúde. In: Schraiber LB, Nemes MIB, Mendes-Gonçalves $\mathrm{RB}$, organizadores. Saúde do adulto: programas e ações na unidade básica. São Paulo: Editora Hucitec; 1996. p. 251-61.

Recebido em 18/Out/2004

Versão final reapresentada em 05/Abr/2005 Aprovado em 18/Mai/2005 